\title{
Comparison of Two High-Intensity Interval Training (HIIT) for Two Weeks on Fat Oxidation, Body Fat Percentage and $\mathrm{Vo}_{2 \max }$ in Overweight Young Men
}

\author{
Mahdi Gholizadeh ${ }^{1 *}$, Mohammadreza Kordi ${ }^{2}$ and Ali Akbarnejad ${ }^{2}$ \\ ${ }^{1}$ Department of Physical Education and Sport Sciences, Guilan University, Iran \\ ${ }^{2}$ Department of Physical Education and Sport Sciences, Tehran University, Iran \\ Submission: June 01, 2018; Published: June 08, 2018 \\ *Corresponding author: Mahdi Gholizadeh, Department of Physical Education and Sport Sciences, Guilan University, Rasht, Iran, \\ Tel: 989142855528; Email: m.qolizadeh66@gmail.com
}

\begin{abstract}
Introduction: The purpose of this study was to Comparison the effects of six sessions high intensity interval training (HIIT) on fat oxidation and $\mathrm{VO}_{2 \max }$ and body fat percentage in sedentary overweight young men.

Methods: Sixteen inactive overweight young men voluntarily participated in this study and randomly divided into two Groups: Group A (n: 8, age: $24.21 \pm 2.01$, height: $174.22 \pm 3.91$, weight: $79.27 \pm 7.59$, BMI: $26 / 54 \pm 2 / 6$ ) and Group B (n: 8, age: $24.23 \pm 1.41$, height: $176.22 \pm 3.88$, weight: 81.27 \pm 6.23 , BMI: $26 / 32 \pm 2 / 9)$. Two groups performed three HIIT sessions per week for two weeks. Group A ( $4 \times 1 \mathrm{~min} / 4 \mathrm{~min}$ R) and Group B $(4 \times 30 \mathrm{~s} / 2 \mathrm{~min} \mathrm{R})$ performed maximal sprint running within a $20 \mathrm{~m}$ area. Respiratory gas was collected 24 hours before and after exercise protocol in fasting state. Data were analyzed by dependent $\mathrm{t}$ test for between groups and paired $\mathrm{T}$ test for within groups.
\end{abstract}

Results: Statistical analysis showed that, Six sessions of HIIT, lead to increasing $43 \%$ of fatty oxidation in Group A in during exercise test, that these changes were significant compared to the Group B (with increasing $11 / 5 \%$ fatty oxidation).changes in weight and present of body fat were significant only in group A. present study showed, a significant increase in $\mathrm{VO}_{2 \max }$ in Group A (5/19\%) and Group B (4\%).

Conclusion: According to the results of this study, it appears six sessions HIIT lead to improvement of fatty oxidation and store glycogen. In conclusion, it appears that HIIT In addition to reduce the body fat and increase the aerobic fitness, is a time efficient means for decrease weight and improving oxidation capacity in sedentary overweight young men.

Keywords: High Intensity Interval Training; Fat Oxidation; $\mathrm{VO}_{2 \max }$; Body Fat Percent

\section{Introduction}

Overweight and obesity are associated with an increased risk of coronary heart disease, stroke, hypertension, type 2 diabetes and certain specific cancers, with body mass index of more than $25 \mathrm{~kg} / \mathrm{m} 2$ increasing insulin resistance, Excessive insulin levels in the blood and high blood sugar, leading to an increased risk of diabetes up to 60 times in women and 42 times in men [1,2]. In recent years, metabolic syndrome has become mild to severe in metabolic syndrome in increasingly developing societies, in Iran, the published statistics show an increase of $70 \%$ in overweight among adolescents [3]. According to the World Health Organization, 1.6 billion adults (over 15) are overweight in the world, and at least 400 million of them are overweight. Men aged 8 to 10 years are less likely to live than normal, and one out of every three in the world is overweight and one in ten is obese. According to statistics, more than 2.3 billion people in the world are expected to become overweight by 2015 [4].
In order to improve the physical condition of obese and diabetic people, instead of medication, sporting activities, including aerobic and aerobic exercises, are used, each of which, through various mechanisms, affects the improvement of the physical condition [5]. Today, HIIT is used in place of traditional endurance training due to its attractiveness, diversity, and metabolic compatibility. Many researchers have suggested that HIIT exercises for weight loss are more beneficial than uniform continuity exercises; recent studies have explicitly stated that the implementation of HIIT for fat loss has a better performance than endurance training [6]. Despite the potential health benefits of endurance training, many adults do not participate in these exercises due to lack of adequate time as an important barrier. On the other hand, it has recently been reported that sustainedintensity exercise for 30 minutes with moderate intensity on most days of the week ( 5 days a week) leads to a decrease or 
reduction in fat less than HIIT implementation, which This demonstrates the high performance of HIIT to increase fat oxidation and reduce adipose tissue $[7,8]$.

Trap et al. [8] reported that 15 weeks of HIIT showed a significant decrease in the percentage of total body fat, subcutaneous fat, trunk, and insulin resistance in young women, as compared to the consistent repeat of exercise activity. Future review papers suggested in this regard, future studies in the field of HIIT should compare the type and severity of the nature of this workable practice to specialize it for specific groups [9]. Regarding the available information, the positive effect of HIIT implementation and the lack of similar research on the effects of its different volumes on the reduction of body fat and the study of changes in oxidation of the substrate during its implementation is necessary. Today, most researchers have come to the conclusion that HIIT implementation is the best type of exercise for health and fat loss [10].

But so far, the best type, severity and duration of HIIT for weight loss has not been determined, and there is still a lot of question in this regard. Regarding diversity, low cost of time, metabolic effects similar to endurance sports and more motivation for people to participate in HIIT instead of traditional endurance exercises for healthy and well-balanced combination, identify the effects of this exercise on biochemical changes associated with Obesity and cardiovascular disease are important. According to the presented questions, the research question is to determine which two types of high-frequency periodic exercise (HIIT) lasts for two weeks on fat oxidation, body fat percentage and $\mathrm{VO}_{2 \max }$ in young men Does it have overweight?

\section{Materials and Methods}

The research method used in this research is semiexperimental. Based on the degree of supervision and degree of control, it is a type of laboratory research. The statistical population of this study was all overweight inactive young men (BMI ranging between 25-29) who were residing in the University of Tehran College with the age range of 23-27 years old. In any regular exercise program at least six months before the start of the study. They did not attend; they only did their daily activities. From this society, 16 subjects were voluntarily selected through questionnaires and randomly divided into two groups of HIIT. A table of attributes has been given to their subjects. Subjects were divided into two groups: 1-HIT (4 active 1-minute activity with maximum intensity and 4 minutes of inactive rest) and HIIT-2 (4 active 30 seconds with maximum intensity and 2 minutes passive rest), a 20-meter distance It was characterized by three cones for two weeks and three sessions each week at 18-20 hours each week. Before the start of the training protocol, each participant had 5 minutes of warm-up program for 5 minutes, and at the end of each training session they were allowed to cool down for 5 minutes. It should be noted that this protocol a validated test is for the evaluation of the efflux function [6]. 24 hours before the first training session and 24 hours after the last session, all subjects in the fasting state (at 8:30am) were presented in the laboratory for two separate days (Table 1).

Table 1: Descriptive statistics of subjects' individual characteristics (mean \pm standard deviation).

\begin{tabular}{|c|c|c|c|c|}
\hline Variables & \multicolumn{2}{|c|}{ HIT-1 } & \multicolumn{2}{c|}{ HIT-2 } \\
\hline & PER & POST & $24.17 \pm 2.15$ & POST \\
\hline Age $(\mathrm{Y})$ & $24.21 \pm 2.01$ & - & $176.21 \pm 3.83$ & - \\
\hline Height(cm) & $174.13 \pm 3.41$ & - & $81.43 \pm 2.36$ & $80.25 \pm 3.01$ \\
\hline Weight(kg) & $79.64 \pm 1.28$ & $78.11 \pm 1.38^{*}$ & $26.32 \pm 1.3$ & $26.18 \pm 1.57$ \\
\hline BMI(kg/m2) & $26.54 \pm 1.4$ & $25.85 \pm 1.59$ & $26.52 \pm 1.79$ & $26.11 \pm 2.38$ \\
\hline Body fat (\%) & $25.72 \pm 2.53$ & $24.12 \pm 2.33^{*}$ & & - \\
\hline
\end{tabular}

After 10 minutes warming, Bruce test was done. An analyzer gas analyzer (Quark b2 in Italy) was used to analyze respiratory gases. For weight measurement, subjects with light clothing were standing on a scale and their weight was recorded in kilograms. To measure the body fat percentage, subcutaneous fat thickness measurement at three points of the chest, abdomen and thigh was performed using Skin Fold Harpenden caliper, RH15 9LB model, with a precision of 0.2 millimeters in England. All measurements are carried out on the right side of the body. After measuring the thickness of subcutaneous fat in the chest, abdomen and thigh, the percentage of fat was calculated using Jackson Pollack's general formula. To calculate the fat oxidation rate, Fern's formula [11] was used:

$1.67 X V O 2(L / \mathrm{min})-1.67 X V O C 2(L / \mathrm{min}):$ fatoxidation $(\mathrm{g} / \mathrm{min})$

\section{Data Analysis}

Kolmogorov-Smirnov test to determine the normality of the data and given that the results of the test data showed normal distribution, the parametric statistical tests were used. In order to investigate the changes between groups, independent t-test and intra-group paired t-test were used. The collected data were analyzed by SPSS-18 software. For all statistical tests, the significance level was considered as $\alpha=0.05$.

\section{Results}

Table 2 shows that the HIIT intervention in the HIIT-1 group had an increase in fat oxidation $43 \%$ and $\mathrm{VO}_{2 \max } 19.5 \%$, as well as a body fat percentage $4.18 \%$. In the group (HIIT-2) fat oxidation increased by 11.5 percent, $\mathrm{VO}_{2 \max }$ increased by 4 percent body fat 
decreased by 1.04 percent. Results showed statistical significance only in the group HIIT-1 also significant between the two groups respectively. Results of independent $t$ test showed that average fat oxidation there is a significant difference between the two groups after the intervention (Table 3 ).

Table 2: Paired t-test results for the oxidation of fat and percentage of body fat and $\mathrm{VO}_{2 \max }$ in Group $1 \mathrm{HIIT}$ - and 2 -HIIT before and after the test.

\begin{tabular}{|c|c|c|c|c|c|c|c|c|}
\hline Variables & \multicolumn{2}{|c|}{ T Value } & \multicolumn{2}{c|}{ P } & \multicolumn{2}{c|}{ Mean Difference } & \multicolumn{2}{c|}{ Percentage of Changes } \\
\hline & HIT-1 & HIT-2 & HIT-1 & HIT-2 & HIT-1 & HIT-2 & HIT-1 & HIT-2 \\
\hline $\begin{array}{c}\text { Fat Oxidation } \\
\text { (g/min) }\end{array}$ & -11 & -3.8 & $0 / 00^{*}$ & 0.06 & 0.027 & -0.021 & +43 & +11.5 \\
\hline $\begin{array}{c}\mathrm{Vo}_{2 \max } \\
\begin{array}{c}\text { Body Fat } \\
\text { Percentage }\end{array}\end{array}$ & -3.3 & -3.2 & $0.01^{*}$ & $0.01^{*}$ & 2 & -1.5 & +5 & +4 \\
\hline
\end{tabular}

Table 3: Results of independent t-test showed that the average $\mathrm{VO}_{2 \max }$ there is a significant difference between the two groups after the exercise intervention $(P=0.05)$.

\begin{tabular}{|c|c|c|c|c|c|c|c|}
\hline \multirow[t]{2}{*}{$\mathbf{P}$} & \multirow[t]{2}{*}{$\mathbf{t}$} & \multirow[t]{2}{*}{$\begin{array}{c}\text { Mean } \\
\text { Difference } \\
\end{array}$} & \multirow[t]{2}{*}{ df } & \multicolumn{2}{|c|}{ SD \pm Mean } & \multirow[t]{2}{*}{ Groups } & \multirow[t]{2}{*}{ Variables } \\
\hline & & & & Post Training & Pre Training & & \\
\hline \multirow{2}{*}{$0.05^{*}$} & \multirow{2}{*}{2.05} & \multirow{2}{*}{2.125} & \multirow{2}{*}{14} & $38.76 \pm 2.46$ & $36.37 \pm 2.13$ & HIT-1 & \multirow{2}{*}{$\mathrm{Vo}_{2 \max }$} \\
\hline & & & & $37.45 \pm 2.81$ & $35.87 \pm 3.11$ & HIT-2 & \\
\hline
\end{tabular}

\section{Discussion and Conclusion}

The present study showed that HIIT treatment in the group (HIIT-1) showed significant changes in fat oxidation and $\mathrm{VO}_{2 \max }$ as well as body fat percentage. In the HIIT-2 group, lipid oxidation increased by $11.5 \%, \mathrm{VO}_{2 \max }$ increased by $4 \%$, body fat decreased by $1.04 \%$, and only fat oxidation was significant. Also in Table 4 independent $t$ test results showed that the average fat oxidation there is a significant difference between the two groups after the intervention.

Table 4: Independent t-test to determine the difference between fat oxidation levels after exercise intervention.

\begin{tabular}{|c|c|c|c|c|c|c|}
\hline \multicolumn{2}{|c|}{ Percentage of Changes } & \multirow{2}{*}{$\mathbf{P}$} & \multirow{2}{*}{$\mathbf{t}$} & \multirow{2}{*}{ Mean Difference } & \multirow{2}{*}{ df } & \multirow{2}{*}{ Variables } \\
\hline HIT-1 & HIT-2 & & & & & \\
\hline $11.5 \% \uparrow$ & $43 \% \uparrow$ & $0.00^{*}$ & 12.76 & 0.32 & 14 & Fat Oxidation \\
\hline
\end{tabular}

Research in this area suggests that increased fat oxidation after HIIT may be due to the need for energy to reverse $\mathrm{H}+$ and re-synthesize glycogen and increase epinephrine, grows hormone, and increase energy consumption. Christopher et al. [5] investigate the effects of six weeks HIIT on the capacity of the metabolism of fats and carbohydrates, and reported, this exercise can increase 18 to 29 percent contain several mitochondrial protein (citrate synthase, beta acyl-coenzyme A dehydrogenase and pyruvate dehydrogenase). It also increases the fatty acid transferability (FAT / CD36, FAB Ppm). Therefore, HIIT increases the mitochondrial enzymes and fatty acid transporters in the short term and increases the oxidation of fat $[12,13]$. Talanin et al. [11] reported an increase in fat oxidation after two weeks of HIIT ( 4 minutes of activity with 2 minutes of rest).

Molecular mechanisms affecting skeletal muscle adaptation through HIIT exercises have been somewhat investigated; research suggests that activity intensity is a key factor in increasing PGC1- $\alpha 2$ (major enzyme in mitochondria biogenesis), and Gibala et al. [14] and Little et al. [15] reported a significant increase in PGC1- $\alpha$ three hours after the HIIT. Upstream signals that stimulate mitochondrial PGC1- $\alpha$ are not well known.
Probably to changes in the strong potential phosphorylation (ATP / ADP AMP) to the activity concerned, and also Chen et al. [16] activation of AMPK, Gibala et al. [14] activation of P38 / MAPK possibly through increased production of ROS and Kang et al. [17] increased PGC1- $\alpha$, have shown that mitochondrial biogenesis ; And Little et al. [15] found that two-week HIIT (10×1 min cycling with $1 \mathrm{~min} \mathrm{R}$ ) increased 25 percent PGC1- $\alpha$ [15-18]. Therefore, one of the causes of a $43 \%$ increase in FAT oxidation in the present study may be increased mitochondria.

Increased mitochondrial oxidative enzymes associated with the use of fat as fuel increases. Another possible mechanism for increased fat oxidation increased hormones respectively. As reported in Table 4, the amount of fat oxidation was higher in the group HIIT-1 that-be due to the type of HIIT-1 and hormone response to intensity. Increasing catecholamines during HIIT is an important feature of this type of activity, especially epinephrine, which causes lipolysis and is the main responsible for the release of FFA from adipose tissue.

Growth hormone response to HIIT is considerable. After HIIT the concentration of this hormone is 10 times higher than 
the rest, which increases lipolysis. During the HIIT, despite the increase in blood lactate, the FFA transfer also increased and the amount of glycerol released from fat was also increased [6]. The increase in glycerol in the HIIT group supports the fact that HIIT results in more acid-fat transfer and oxidation. Talanin et al. [11] has reported a $13 \%$ increase in plasma epinephrine and $36 \%$ fat oxidation after two weeks of HIIT.

The hormones produced from adipose tissue play a vital role in regulating and consuming energy and metabolism of fat and carbohydrates. The mechanisms and function of adipocytokines, such as adiponectin, leptin, resistin, IL- 6 , and TNF- $\alpha$, in skeletal muscle, regulate the production and consumption of energy. Overall, lipid oxidation markers have significantly changed after six sessions of HIIT. Overall, fat oxidation markers have significantly changed after six sessions of HIIT. The most important changes in biochemistry are the changes in the amount and activity of various enzymes involved in exercise, the implementation of HIT increases the activity of both aerobic and anaerobic enzymes. McDougall et al. [19] reported a significant increase in the activity of hexokinase, phosphorus, citrate synthase, suction dehydrogenase and malate dehydrogenase enzymes after seven weeks of HIIT implementation, and concluded that the implementation of HIIT can increase both oxidative and glycolic enzymes [20].

It has been reported thataerobicfitnessincreasessignificantly after the minimum HIIT implementation. White et al. [21] conducted a two-week intervention of HIIT implementation with three sessions per week, including four to six Wingate tests with four minutes of recovery time [7]. In this study, untrained men $\mathrm{VO}_{2 \max }$ by as much as seven percent increased. A $13 \%$ increase in $\mathrm{VO}_{2 \max }$ has also been reported following a HIIT program for two weeks [11]. HIIT protocols increase $\mathrm{VO}_{2 \max }$ by four percent [20] and six to eight percent [22] for six to eight weeks. HIIT implementation of Wingate for 12 to 24 weeks (long-term) large increase in $\mathrm{VO}_{2 \max } 41$ and 46 percent of patients with type II diabetes and cardiac rehabilitation made [23]. Protocols with a lower intensity ( 8 seconds of activity / 12 seconds of recovery) with extended periods of more than 15 and 12 weeks resulted in an increase of $24 \%$ [24] in VO2 max. Overall, these results show that the implementation of different forms of HIIT by young adults and elderly patients for two to 15 weeks results in a significant increase in VO2 max between four and 46 percent. The present study showed that the HIIT-1 exercise protocol $(4 \times$ 1 min activity with high speed and 4 minute rest) caused more compatibility in oxidative capacity, increased $\mathrm{VO} 2$ max, weight loss compared to HIIT- $2(4 \times 30$ s activity with 2 minutes Rest). It is recommended that overweight individuals use a HIIT-1 group protocol to reduce weight and increase muscle capacity for fat oxidation.

\section{Acknowledgment}

Of all the participants in this research who as a subject participated until the last stage, I appreciate and thank Dr.
Mohammad Reza Kurdi, the professor of medicine and the director of the lab of Tehran Physical Education Faculty.

\section{References}

1. Stevens J, Cai J, Steensberg ER, Williamson DF, Thun MJ (1998) The effect of age on the association between body-mass index and mortality. Journal of Applied Physiology 338(1): 1-7.

2. Rose A, Richter A (2008) Obesity as a disease. Eur j Appl physiol 53: 307-321.

3. Azizi F, Etemadi A, Salehi P, Zahedi S (2003) Prevalence of metabolic syndrome in an urban population: Tehran lipid and glucose study. Tehran university medical journal 65(1): 389-399.

4. World health organization recommendation for obesity (2012) obesity update.

5. Christopher GR, Perry JF (2008) High-intensity aerobic interval training increases fat and carbohydrate metabolic capacities in human skeletal muscle. Applied physiology, nutrition, and metabolism 33(6): 1112-1897.

6. Martin G, Jonathan P, Maureen J, MacDonald M, John A (2012) Physiological adaptations to low-volume, high-intensity interval training in health and disease. J physiol 590(5): 1077-1108.

7. Dunn S, Lunde IG, Anton SL, Bruusgaard JC, Rana ZA (2009) Effects of exercise and dietary intervention on metabolic syndrome markers of inactive premenopausal women. metabolism clinical and experimental 670: $1134-1115$.

8. Trapp EG, Chisholm DJ, Freund J, Boutcher SH (2008) Effects of highintensity interval exercise training on fat loss and fasting insulin levels of young women. International journal of obesity 32(4): 684-691.

9. Stephen H Boutcher (2011) High-intensity intermittent exercise and fat loss. Journal of obesity 868: 23-31.

10. Mcmurray RG, Andersen B (2009) The influence of exercise on metabolic syndrome in youth: A review. Am J lifestyle med 4(2): 176186.

11. Talanian J, Galloway S, Heigenhauser F, Bonen A (2007) Two weeks of high-intensity aerobic interval training increases the capacity for fat oxidation during exercise in women. J appl physio 102(4): 176-185.

12. Burgomaster K, Howarth R, Phillips SM, Rakobowchuk M, Macdonald J, et al. (2008) Similar metabolic adaptations during exercise after low volume sprint interval and traditional endurance training in humans. J physiol 586(1): 151-160.

13. Gibala M, Little J, Vatson M, Wilkin GP, Burgomaster K, Safdar A (2006) Short-term sprint interval versus traditional endurance training: similar initial adaptations in human skeletal muscle and exercise performance. J physiol 575(3): 901-911.

14. Gibala M, Sean L, McGee P, Kirsten F (2009) Brief intense interval exercise activates AMPK and p38 MAPK signaling and increases the expression of PGC-1in human skeletal muscle. Applied physiology, nutrition, and metabolism 106(3): 929-934.

15. Little J, Taylor CT, Bishop D, Tarnopolsky M, Gibala M (2011) An acute bout of high-intensity interval training increases the nuclear abundance of pgc- $1 \alpha$ and activates mitochondrial biogenesis in human skeletal muscle. J physiol 300(6): 1303-1310.

16. Chen P, Mcconell G, Michell B, Snow R, Canny B (2000) AMPK signaling in contracting human skeletal muscle. Am j physiol endocrinol metab 279(5): 1202-1206.

17. Kang C, Moore K, Dickman J (2009) Exercise activation of muscle peroxisome proliferator-activated receptor- $\gamma$ coactivator- $1 \alpha$ signaling is redox sensitive. Free bio med 47(10): 1394-1400. 
18. Little J, Wilkin GP, Gibala M (2011) A practical model of low-volume high-intensity interval training induces mitochondrial biogenesis in human. The Journal of physiology 342: 1303-1310.

19. Macdougall JD, Hicks A, Macdonald JR, Mckelvie S, Green H (1998) Muscle performance and enzymatic adaptations to sprint interval training. J appl Physiol 84(6): 2138-2142.

20. Whyte J, Gill M, Cathcart AJ (2010) Effect of 2 weeks of sprint interval training on health-related outcomes in sedentary overweight/obese men. Metabolism clinical and experimental 59(10): 1421-1428.

21. Mclure SA, Summerbell CD, Reilly J (2009) Objectively measured habitual physical activity in a highly obesogenic environment. Child care health dev 35(3): 369-375.
22. Helgerud J, Hydal k, wang E (2007) Aerobic high-intensity intervals improve $\mathrm{vo}_{2 \max }$ more than moderate training. Medicine and science in sports and exercise 39(4): 665-671.

23. Mourier A, gautier F, Kerviler (1997) Mobilization of visceral adipose tissue related to the improvement in insulin sensitivity in response to physical training: Effects of branched-chain amino acid supplements. Diabetes care 20(3): 385-391.

24. Jonathan P, Little A, Wilkin M, Mark A, Martin J (2010) A practical model of low-volume high-intensity interval training induces mitochondrial biogenesis in human skeletal muscle: Potential mechanisms. J Physiol 588(6): 1011-1022.

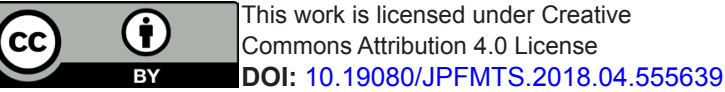

